\title{
PROCUREMENT 4.0: COMO A APLICAÇÃO DE NOVAS TECNOLOGIAS E ROBOTIZAÇÃO TRANSFORMARÁ A ÁREA DE SUPRIMENTOS DAS EMPRESAS
}

\author{
Spec. Paulo Henrique Domingues Costa ${ }^{1}$ \\ paulohdcosta@hotmail.com \\ Prof. M. Sc. Rickardo Léo Ramos Gomes ${ }^{2}$ \\ rickardolrg@yahoo.com.br \\ https://orcid.org/0000-0001-6101-9571
}

Para citar este artículo puede utilizar el siguiente formato:

Paulo Henrique Domingues Costa y Rickardo Léo Ramos Gomes: "Procurement 4.0: como a aplicação de novas tecnologias e robotização transformara a área de suprimentos das empresas", Revista Caribeña de Ciencias Sociales (vol 10, № 7 julio-septiembre 2021, pp. 25-37. En línea: https://www.eumed.net/es/revistas/caribena/julio-septiembre-2021/novas-tecnologias-empresas

\section{RESUMO}

As empresas transformam-se, atualmente, através da inserção da robotização e inteligências artificiais nos seus processos fabris de forma geral. Esta evolução relaciona-se à chamada Indústria 4.0 e à estratégia Procurement 4.0 que visam garantir agilidade em processos de compra e de abastecimento. Este artigo tem como objetivo geral demonstrar a importância da adoção, por parte das empresas, do Procurement 4.0 de modo a garantir evolução nos processos empresariais. Estes são os objetivos específicos: Esclarecer o conceito de Procurement 4.0; Explicar as ferramentas que poderão ser utilizadas a partir da introdução do conceito e da aplicação de Procurement 4.0; Destacar os benefícios deste conceito. A metodologia empregada nesta pesquisa tem caráter descritivo e exploratório, com intuito de observar e reproduzir as ideias centrais acerca do assunto principal proposto e servir de base para instigar o aprofundamento do tema por profissionais e acadêmicos da área. Conclui-se que as empresas devem continuar atendendo os anseios do mercado consumidor e, também, devem permanecer competitivas, promovendo a modernização

1 Graduado em Engenharia de Produção pelo Centro Universitário do Leste de Minas Gerais; Especialista em Gestão Estratégica de Empresas pela Faculdade Pitágoras; Pós-graduando em Gestão de Suprimentos pelo Instituto Euvaldo Lodi; Especialista de Suprimentos na Companhia Siderúrgica do Pecém.

2 Prof. da Disc. de Met. Científica (Orientador) - Inst. Euvaldo Lodi; C. U. UniAteneu; C. U. Farias Brito; M. Sc. em Fitotecnia UFC; Spec. em Met. do Ens. de Ciências - UECe; Grad. em Agronomia - UFC; Lic. na Área de Ciências da Natureza, Matemática e suas Tecnologias - UVA; Aperf. em Líderes de Aprendizagem pela Univ.de Harvard; Aperf. em Gestão de Riscos em Projetos - BID; Aperf. em Met. do Trabalho Científico - FIOCRUZ. Curso Aperf. Rastreamento do Contato da COVID-19 Johns Hopkins University; Consultor Internacional (BIRD). Conveniado com a ABNT. Fundador da RLRG Consultoria Científica. 
através da aplicação dos conceitos de Indústria 4.0 e Procurement 4.0 tornando seus processos empresariais mais ágeis através do uso de $\mathrm{Al}$, loT e robotização.

Palavras-chave: Procurement 4.0. Desenvolvimento tecnológico. Novas tecnologias.

\section{PROCUREMENT 4.0: CÓMO LA APLICACIÓN DE NUEVAS TECNOLOGÍAS Y LA ROBOTIZACIÓN TRANSFORMARÁN EL ÁREA DE ABASTECIMIENTO DEL NEGOCIO}

\section{RESUMEN}

Actualmente, las empresas se transforman mediante la inserción de la robotización y la inteligencia artificial en sus procesos de fabricación en general. Esta evolución está relacionada con la denominada Industria 4.0 y la estrategia Procurement 4.0, que tienen como objetivo asegurar la agilidad en los procesos de compras y aprovisionamiento. Este artículo tiene como objetivo demostrar la importancia de la adopción, por parte de las empresas, de Procurement 4.0 para asegurar la evolución de los procesos de negocio. Estos son los objetivos específicos: Aclarar el concepto de Adquisiciones 4.0; Explicar las herramientas que se pueden utilizar desde la introducción del concepto y aplicación de Adquisiciones 4.0; Destaque los beneficios de este concepto. La metodología utilizada en esta investigación tiene un carácter descriptivo y exploratorio, con el fin de observar y reproducir las ideas centrales sobre el tema principal propuesto y servir de base para impulsar la profundización del tema por parte de profesionales y académicos del área. Se concluye que las empresas deben seguir satisfaciendo las necesidades del mercado consumidor y, además, deben seguir siendo competitivas, impulsando la modernización a través de la aplicación de los conceptos de Industria 4.0 y Procurement 4.0, agilizando sus procesos de negocio mediante el uso de IA, IoT y robotización.

Palabras clave: Adquisiciones 4.0. Desarrollo tecnológico. Nuevas tecnologías.

\section{PROCUREMENT 4.0: HOW THE APPLICATION OF NEW TECHNOLOGIES AND ROBOTIZATION WILL TRANSFORM THE BUSINESS SUPPLY AREA}

\section{ABSTRACT}

Companies are currently transformed through the insertion of robotization and artificial intelligence in their manufacturing processes in general. This evolution is related to the so-called Industry 4.0 and the Procurement 4.0 strategy, which aim to ensure agility in purchasing and supply processes. This article aims to demonstrate the importance of the adoption, by companies, of Procurement 4.0 in order to ensure evolution in business processes. These are the specific objectives: Clarify the concept of Procurement 4.0; Explain the tools that can be used from the introduction of the concept and application of Procurement 4.0; Highlight the benefits of this concept. The methodology used in this research has a descriptive and exploratory character, in order to observe and reproduce the central 
ideas about the main subject proposed and serve as a basis for instigating the deepening of the theme by professionals and academics in the area. It is concluded that companies must continue to meet the needs of the consumer market and, also, must remain competitive, promoting modernization through the application of the concepts of Industry 4.0 and Procurement 4.0, making their business processes more agile through the use of $\mathrm{Al}$, loT and robotization.

Subject Descriptor University of Derby: Managerial \& Capability Support, Technology Levers, Process Performance Requirements and Strategic Outcomes.

Keywords: Procurement 4.0. Technological development. New technologies.

\section{INTRODUÇÃO}

Nos dias atuais as organizações têm enfrentado mudanças impactantes na forma de conduzir os negócios e produções industriais, pois a globalização e a troca de dados e informações, de forma instantânea, permitem que todas as empresas tenham acesso rápido às melhores práticas empresariais e, portanto, a competitividade vem se tornando cada vez mais acirrada (Schreiber et al, 2016).

Na outra ponta, os clientes consumidores também têm usufruído de novas tecnologias e do modelo globalizado de consumo, que permite um conhecimento maior de fornecedores e de potenciais fornecedores, bem como de novos produtos e possíveis substitutos aos atuais, muitas vezes mais baratos e mais eficientes. Tudo isso através de plataformas digitais que permitem muito mais comodidade, o resultado disso são clientes muito mais exigentes (Schreiber et al, 2016).

A competitividade do mercado, bem como as exigências dos clientes faz com que as organizações tenham que buscar novas formas de desenvolver o trabalho de forma que aumente a produtividade, tenha flexibilidade para fazer produtos personalizados, mas, ao mesmo tempo, em escala industrial e para isso o tempo e a assertividade nos processos são essenciais.

Entra em cena a nova revolução industrial chamada Indústria 4.0 e que já está bem orientada. As novas tecnologias têm sido amplamente debatidas, estão sendo inseridas cada vez mais nas organizações e remodelando a forma como os trabalhos têm sido desenvolvidos.

Os empregadores dessa nova prática visam desenvolver esse conceito de Indústria 4.0 na produção e nos processos auxiliares, como marketing digital, logística e logística reversa e suprimentos, sendo esta última um processo-chave para garantir maior resultado competitivo financeiro e agilidade para os processos fabris.

Este artigo procura adentrar no conceito de Indústria 4.0 pela perspectiva da área de suprimentos, através do conceito de Procurement 4.0. Tem como objetivo geral abordar a evolução da área, até o momento atual vivido pela globalização, apresentando exemplos onde já é possível 
perceber o emprego deste conceito e o impacto que a utilização deste proporciona às organizações (Schreiber et al, 2016).

Os objetivos específicos são os seguintes: Esclarecer como o conceito de Procurement 4.0 foi desenvolvido a partir do entendimento do conceito da Indústria 4.0; Explicar as ferramentas que poderão ser utilizadas no aprimoramento das funções de suprimentos a partir da introdução do conceito e da aplicação de Procurement 4.0; Destacar os benefícios deste conceito, após a aplicação das ferramentas e promoção do desenvolvimento tecnológico diante desta nova era de desenvolvimento.

A metodologia empregada nesta pesquisa tem caráter descritivo e exploratório, com intuito de observar e reproduzir as ideias centrais acerca do assunto principal proposto e servir de base para instigar o aprofundamento do tema por profissionais e acadêmicos da área.

O Procurement 4.0 é essencial para o desenvolvimento das empresas que buscam progredir no novo conceito de Indústria 4.0, onde a evolução tecnológica aponta, a cada dia, como as empresas podem ser mais eficazes e mais eficientes em um mercado cada vez mais competitivo.

Não diferente disso a área de suprimentos deve procurar automatizar os processos para que todo sistema execute de forma inteligente as melhores escolhas para processos comuns, liberando os profissionais da área executar tarefas que proporcione maiores ganhos financeiros e de produtividade para as empresas.

As máquinas fazem trabalhos de forma inteligente para que os profissionais tenham tempo para fazer trabalhos inteligentes.

Observa-se, finalmente, que muitas empresas ainda não se atinaram para este novo momento industrial tecnológico, motivo pelo qual este tema foi escolhido para ser abordado. $\mathrm{O}$ artigo seguiu os seguintes passos estruturais: inicia-se com a introdução, contextualizando o tema do artigo no intuito de apresentar o que está sendo abordado.

Em seguida foi elaborado, com base nas literaturas atualmente disponíveis, a fundamentação teórica. Apresenta-se na sequência a metodologia utilizada para a execução do artigo e finaliza-se com as considerações finais a fim de intenta apresentar ao leitor o possível tirocínio do tema abordado.

A evolução constante dos processos industriais é visível e inevitável e, neste momento, o mundo passa por essa evolução chamada Indústria $4.0 \mathrm{com}$ a aplicação de robôs e sistemas capazes de realizar atividades de forma independente e inteligente.

\section{FUNDAMENTAÇÃO TEÓRICA}


Neste artigo, foram levantadas informações sobre a origem do conceito iniciado a partir da Indústria 4.0 e quais foram as evoluções para chegar ao Procurement 4.0.

O estudo tem intuito de trazer um novo conceito de supply chain que possibilita agilidade e economia nos processos a partir da utilização das novas tecnologias com intuito principal de gerar maior lucratividade e competitividade às empresas.

\subsection{Indústria 4.0}

Tido como o $4^{\circ}$ grande marco revolucionário industrial, o termo surgiu pela primeira vez na Alemanha em 2011, promovido por acadêmicos, políticos, e representantes empresariais com o ideal de aumentar a competitividade das indústrias da Alemanha (Schwab, 2016, apud Bueno et al 2019a).

Na figura 1 é possível acompanhar a evolução da indústria no decorrer dos últimos 237 anos.

\section{Figura 1}

Da Indústria 1.0 à Indústria 4.0

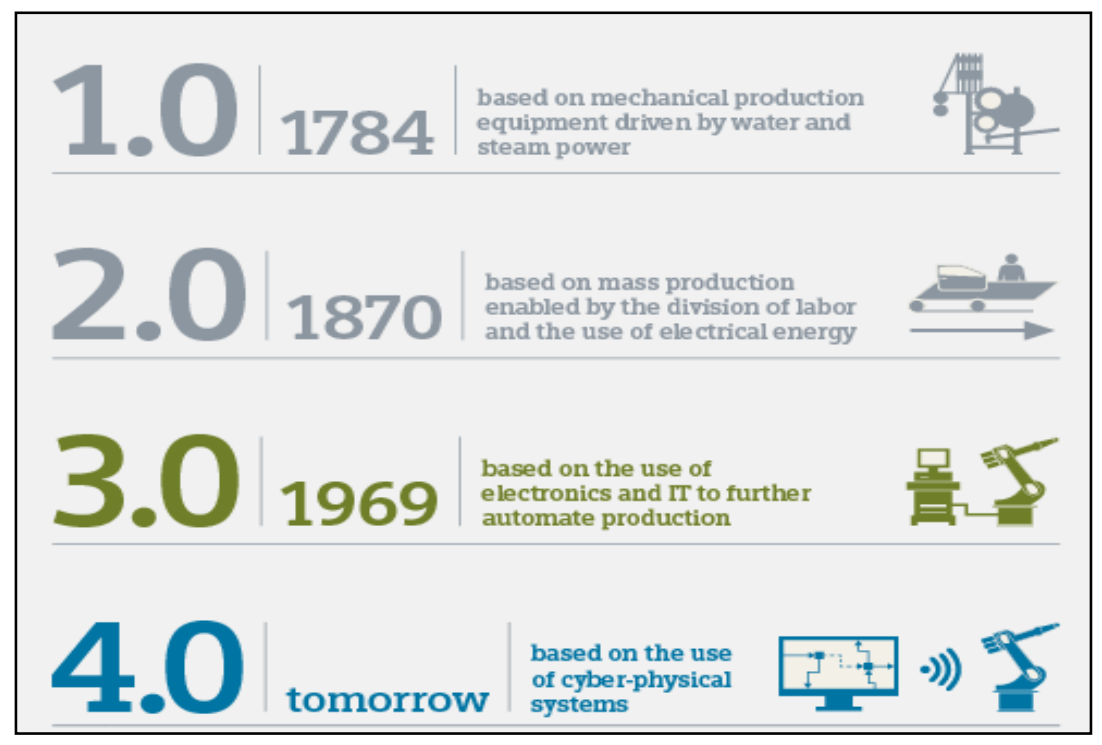

Fonte: Schwab (2016)

Já o Blog Inovação Industrial, publicado por Petronas (2019), atribui o surgimento da quarta revolução industrial através do investimento de países desenvolvidos em novas ferramentas tecnológicas, conforme apresentado na figura 2. 


\section{Figura 2}

A origem da 4⿳亠丷 Revolução Industrial.

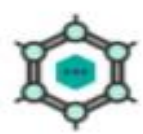

criaçăo do Plano

High-Tech Strategy

2020, na Alemanha,

cujo foco é se tornar

o principal gerador.

integrador e fornecedor

de tecnologias de

produça inteligentes:

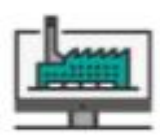

criaçâo do

Programa Advanced

Manufacturing. nos

EUA, cuja ideia gira

em torno da inovaçăo

na manufatura

como gatilho para o

desenvolvimento no país:

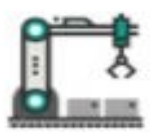

criaçăo de uma

estratégia chinesa.

em 2012, cujo objetivo

é conseguir um

posicionamento entre

as principais forças

industriais no mundo.

Fonte: Petronas (2019)

De acordo com Souza (2018, p. 14) "Indústria 4.0, basicamente, é a descentralização das tomadas de decisões que temos em uma fábrica para que ela aconteça de maneira cada vez mais automática".

Bueno et al (2019a) apontam quatro componentes principais, considerados chaves para a Indústria 4.0: Smart Factory, Internet of Things (IoT), Internet of Service (IoS) e Cyber-Physical Systems.

Hermann et al (2015 apud Bueno et al, 2019a) introduzem estes quatro componentes:

> Cyber-Physical Systems (CPS): Sistemas físicos com sensores controlados de forma autônoma por softwares.

$>$ Internet das coisas (IoT): conectividade entre sistemas físicos, capazes de processar informações virtuais e influenciar atividades nos sistemas físicos.

$>$ Internet de serviços (loS): serviços acessíveis através de tecnologias World Wide Web.

> Smart Factory: fábricas inteligentes que possibilitam processo de produção de produtos independentes e rastreáveis, permitindo produção em massa, porém flexível e individualizada.

De acordo com Schwab (2016, p.37), "A quarta revolução industrial terá um impacto monumental na economia global; será tão vasto e multifacetado que fica difícil separar determinado efeito do outro".

Na Indústria 4.0 é recomendável categorizar as atividades organizacionais para criação de valor, para tanto Procurement 4.0 é uma das atividades de apoio. Levando-se em conta este contexto, avalia-se que medida a função compras, a disciplina e a atividade de aquisição, 
propriamente dita, podem mudar assumindo uma interface mais crítica e estratégica dentro da área de gestão da cadeia de abastecimento, apoiando a eficiência organizacional, eficácia e lucratividade em uma perspectiva de longo prazo (Haddud, 2018).

\subsection{Exemplos da Aplicação do Conceito Indústria 4.0}

Petronas (2019) aponta 9 tecnologias e ferramentas essenciais para que uma empresa possa ser considerada parte da Indústria 4.0: Robôs automatizados; Manufatura aditiva, conhecida como Impressão 3D; Simulação ou Simuladores; Integração Horizontal e Vertical de Sistemas; Internet das coisas, conexão entre objetos físicos e pessoas em uma rede; Big Data; Computação na nuvem; Segurança cibernética e Realidade aumentada.

Schwab (2016, p. 21) apresenta exemplos atuais internacionais onde já é possível identificar empresas que utilizam ferramentas e tecnologias abordadas no conceito de indústria 4.0, e quão escalável é a produção e aumento da competitividade destas empresas:

A escala e o escopo das mudanças explicam por que as rupturas e as inovações atuais são tão significativas. A velocidade da inovação em termos de desenvolvimento e ruptura está mais rápida do que nunca. Os atuais disruptores - Airbnb, Uber, Alibaba e afins - que hoje já são nomes bem familiares, eram relativamente desconhecidos há poucos anos. onipresente iPhone foi lançado em 2007. Mas, no final de 2015, já existiam cerca de 2 bilhões de smartphones. Em 2010, o Google anunciou seu primeiro carro totalmente autônomo. Esses veículos podem, rapidamente, se tornar uma realidade comum nas ruas.

Souza (2018) chama atenção para alguns exemplos brasileiros, como o caso da empresa catarinense Embraco, fabricante de refrigeradores que fundou em parceria com a FIESC, Federação das Indústrias de Santa Catarina, a Associação Brasileira de Internet Industrial para colocar em prática a aplicação do conceito da Indústria 4.0 de forma que o uso das novas tecnologias possa proporcionar o aumento da competitividade.

Souza (2018) ainda cita multinacionais como Embraer, Volkswagen, Ambev e Jeep, sendo essa última considerada uma das indústrias instaladas no Brasil com o maior nível de automação, onde são produzidos 45 carros por hora com trabalhos executados por robôs em quase todo 0 processo produtivo.

\subsection{Procurement 4.0}

Bueno (2019, p. 03) explica sobre a ideia central do termo Procurement e a sua abrangência no contexto corporativo: 
Procurement termo em inglês que também pode ser traduzido como adquirir ou, ainda licitar. Como estamos num ambiente corporativo, no entanto Procurement corresponde a um processo mais amplo que inclui Sourcing, função de compras, incorporando um caráter estratégico, abrangendo as áreas comerciais, gestão de projetos e logística, incluindo a gestão dos estoques, participação no processo produtivo, distribuição e relacionamento com clientes e fornecedores.

Glas e Kleemann (2016, apud Bueno et al, 2019b) descrevem que Procurement 4.0 envolve automação dos processos e digitalização final tanto dentro da companhia quanto em interface com fornecedores, não limitado somente pela aplicação por aplicação de novas tecnologias.

Procurement 4.0 advindo da Indústria 4.0 é a evolução do MRP aos dias atuais tendo avançado em duas dimensões: no grau de integração transversal e funcional e no grau de automatização de sistemas que reduzem o trabalho manual na área de compras. (Kagermann et al., 2014, apud Bueno et al. 2019b).

Os sistemas de informação avançaram, continuamente, desde que todas as etapas produtivas, incluindo aquisições de materiais, matérias primas e serviços, passaram a ser monitoradas pela alta administração, havendo em pouco tempo uma evolução a partir do MRP Materials Requirements Planning e MRPII - Manufacturing Requirements Planning, chegando ao ERP - Enterprising Resource Planning. (Stair, 1999, apud Bueno et al. 2019b).

Pinto (2019) em sua pesquisa, onde a maioria dos respondentes foi constituída de empresas de grande porte, constata que a utilização de Sistemas ERP e do e-Procurement é muito significativa no Brasil, sendo essa utilização superior a $90 \%$ entre as empresas pesquisadas.

E-procurement é parte das chamadas tecnologias da informação. As definições são variadas. Presutti (2003, p. 221), por exemplo, define e-procurement como "uma solução tecnológica que facilita as compras corporativas pelo uso da internet". Para Min e Galle (2003), e-procurement diz respeito as transações "business-to-business" que utilizam o comércio eletrônico para identificar fontes potenciais de suprimentos, comprar bem e serviços, fazer pagamentos e interagir com fornecedores. (Grande et al., 2016, p. 35)

Bueno et al (2019a) aponta uma evolução dos sistemas de compras, iniciando pelo MRP e ERP, passando por e-Procurement até o Procurement 4.0, apontando este último como sendo uma função de gerenciamento de negócios. 


\section{Figura 3 -}

A evolução dos sistemas utilizados pela área de Suprimentos

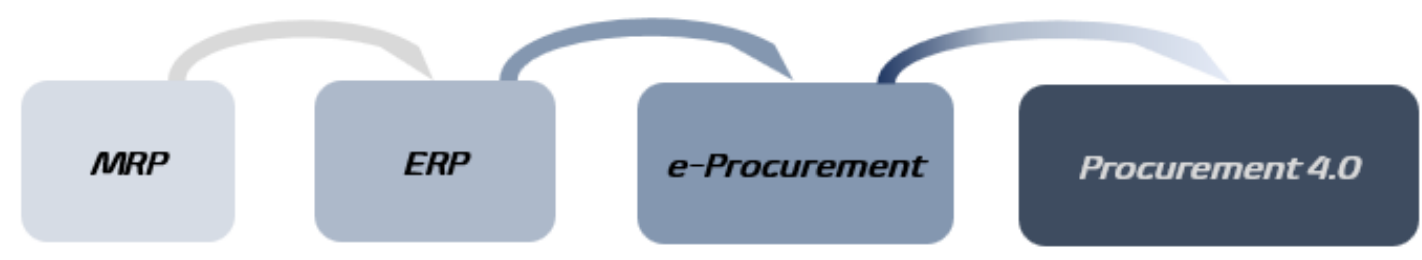

Fonte: Dados do Pesquisador

É possível perceber através das publicações acadêmicas e empresariais uma constante evolução dos sistemas de compras desde o surgimento de uma área específica para tratar as compras e gerenciamento de estoque das empresas, e o momento atual é a transição do eProcurement para o Procurement 4.0.

\subsection{Benefícios da Introdução de Procurement 4.0}

De acordo com Schwab (2016) a nova revolução tecnológica desdobrar-se-á em mudanças socioculturais e econômicas tão grandes que se torna muito difícil de prever quais proporções ela pode chegar.

D. Little (2016, apud Pinto, 2019) aponta que com Procurement 4.0 as decisões de compras não serão baseadas, exclusivamente, em custos e passaram a ser baseadas em valor adicional além do custo, sendo esses valores adicionais gerados através de novos relacionamentos com fornecedores.

Já Batran (2017, apud Pinto, 2019) aponta que com Procurement 4.0 a interface com fornecedor gera cadeia de valor ao negócio, pois é possível mapear do produto final até a matériaprima todas as etapas por onde passou o bem e inclusive é possível checar se há dependência de fornecedores e de propriedade intelectual.

Dessa forma, as inovações são de suma importância para obter diferenciação, e formar uma rede estratégica, tornando-se o cliente predileto e obtendo direitos exclusivos sobre os novos desenvolvimentos de produtos e serviços dos fornecedores.

A PWC - PricewaterhouseCoopers (2016 apud Pinto, 2019) aponta a análise de dados como sendo o ativador mais importante de Procurement 4.0, pois um volume muito grande de dados de fontes diversificadas é processado com a utilização de algoritmos inteligentes das novas tecnologias, podendo com isso compreender as situações de fornecedores e clientes, do mercado e as falhas de equipamentos. 
Para Gracht (2016, apud Bueno, 2019) o paradigma da eficiência e redução de custos está terminando pois os novos conceitos revolucionários propiciam grandes saltos tecnológicos na atualidade, sobretudo na transformação digital.

\subsubsection{Exemplo da aplicação Procurement 4.0}

Um exemplo direto da aplicação do Procurement 4.0, onde as atividades de compras passam a ser executadas de forma inteligente, fisicamente e digitalmente, por meio virtual e sem a interação direta quando o próprio sistema identifica as condições previstas, pode ser observado na pesquisa de Ito e Santos (2020, p.61), que explicam:

Os contratos inteligentes ou smart contracts são instrumentos da tecnologia da informação que encerram os mecanismos da automação do cumprimento integral ou parcial de transações de natureza contratual: o programa possui controle sobre objetos (físicos e digitais) relativos ao cumprimento do contrato e este (ou parcela deste) são escritos em linguagem computacional, no formato de código executável e, uma vez verificada as condições previstas o código é, automaticamente, implementado.

Assim como o exemplo descrito acima, a era da informação possibilita a automatização de processos onde as análises de dados são efetuadas por máquinas e contratos e pedidos são executados a partir dessa análise.

Materiais com consumo regular, a partir do momento que o estoque de matéria prima ou materiais atingem o nível mínimo definido, os sistemas disparam compras através de contratos acordados, tendo sido esses contratos definidos após concorrência ou licitações efetuadas diretamente por máquinas sem intervenções de pessoas em todo o processo de compra. Ou seja, do início ao fim do processo de compra todas as etapas são executadas por computadores sem necessidade de acompanhamento humano.

\section{METODOLOGIA}

A metodologia empregada nesta pesquisa tem caráter descritivo e exploratório, com intuito de observar e reproduzir as ideias centrais acerca do assunto principal proposto e servir de base para instigar o aprofundamento do tema por profissionais e acadêmicos da área.

Os procedimentos técnicos que foram utilizados neste trabalho são classificados como pesquisa bibliográfica desenvolvida a partir de publicações efetuadas à cerca de um tema proposto, sendo assim, tratar-se-á de uma revisão bibliográfica.

Foi realizada uma pesquisa sobre os temas Indústria 4.0 e Procurement 4.0 em livros como 'A Quarta Revolução Industrial' de Schwab (2016), artigos acadêmicos, dois deles publicados por 
Bueno et al (2019a e 2019b), e artigos empresariais como o artigo 'Desvendando a Indústria 4.0' do Blog Inovação Industrial/Petronas (2019), por se tratar de um tema ainda novo e pouco abordado na área acadêmica.

Finalmente, com este estudo foi possível observar que a indústria e o mercado atual passarão por um desenvolvimento com o predomínio das novas tecnologias sendo este um processo irreversível e que garantirá a competitividade das empresas perante o mercado concorrencial, e a não adequação ao novo padrão globalizado será caracterizada pela descontínua evolução tecnológica e pela, consequente, obsolescência empresarial.

\section{CONSIDERAÇÕES FINAIS}

A indústria está em constante transformação desde sua concepção até os dias atuais, iniciando na primeira revolução industrial, com a invenção da máquina a vapor, passando pela segunda e terceira revolução industrial, com produção em massa dos trabalhadores onde a energia elétrica foi principal fator relevante e com a automação de processos produtivos em meados do século XX, culminando nos dias atuais a quarta revolução industrial chamada de Indústria 4.0 e a inteligência das coisas.

Fábricas altamente conectadas, com gerações de dados e armazenamento em nuvens, processamento de dados e tomadas de decisões efetuadas pelas máquinas, tudo para garantir e otimização de processos, velocidade na fabricação de produtos personalizados e com alta qualidade em prazos mínimos, garantem aos dias atuais o atendimento do mercado consumidor.

Toda a conectividade e inteligência dos processos estão inclusos em todas as áreas e etapas do processo fabril. E ponto importante neste quesito está a logística de movimentação, transporte e as compras. Todo o processo de suprimentos de uma indústria agindo de forma inteligente contribui para o sucesso industrial.

É esta etapa que propiciará ganhos para empresa, em tempos onde quem determina o preço do produto é o cliente e o quanto ele está disposto a pagar por um determinado bem, pois minimizar custos garantindo qualidade das matérias primas e MRO é responsabilidade da área de suprimentos. Essa etapa é preponderante para o aumento da margem de lucro da indústria.

Os estudos bibliográficos, aqui desenvolvidos, evidenciam que a aplicação do Procurement 4.0 proporcionará ganho através de custo-benefício e não apenas o menor preço, pois poderão ser utilizadas ferramentas para acompanhar desempenho de fornecedores, variações dos materiais, tempo de vida útil pelo custo, robotização de compras simples necessárias, mas que não agregam valor para as empresas.

Este trabalho apresentou empresas que já se destacam no cenário mundial com a aplicação da Indústria 4.0 e Procurement 4.0 e demonstra, através dos levantamentos bibliográficos muito 
atuais, que o futuro da indústria e o futuro da área de suprimentos passa pela inovação dos usos das novas tecnologias pautadas em robôs, Inteligência Artificial (Al) e Internet das Coisas (loT).

Conclui-se, por fim, que as empresas devem continuar atendendo os anseios do mercado consumidor e, ao mesmo tempo, devem permanecer competitivas perante o mercado concorrente. Assim sendo, as empresas que não aderirem à evolução tecnológica advinda da aplicação da Indústria 4.0 e Procurement 4.0 enfrentarão a possibilidade real de se tornarem obsoletas, já que torna-se caro manter um setor crítico de abastecimento, suprimentos, comprometendo tempo e material humano em processos que podem ser efetuados de forma ágil através do uso de Al, loT e robotização.

Ao final propõe-se três linhas para futuras pesquisas dentro da temática aqui abordada. A primeira voltada para a investigação nos campos internos das empresas com potenciais possibilidades de implantação do Procurement 4.0.

A segunda é pesquisar onde o Procurement 4.0 pode impactar mais, se no setor de manufatura ou no setor de serviços e se ocorrem diferenças ou semelhanças nos subcampos da indústria.

A terceira é a realização de entrevistas com funcionários e gerentes envolvidos no Procurement 4.0, de modo a compartilhar dados, experiências, práticas e insights, além de derivar ideias adequadas de forma a orientar outras organizações.

\section{REFERÊNCIAS}

Bueno, Robson Elias; Bonilla, Sílvia Helena; Fernandes, Samuel; Santos, Helton Almeida Dos; Barbosa, Lorivaldo Rodrigues. (2019a). A Evolução da Logística de Suprimentos: Procurement 4.0. X FATECLOG - Logística 4.0 \& A Sociedade do Conhecimento. Guarulhos: FATEC. ISSN 2357-9684.

Bueno, Robson Elias; Santos, Helton Almeida Dos; Toloi, Rodrigo Carlo; Mosca, Sílvia Helena Bonilla. (2019b). Procurement 4.0: Impactos, Oportunidades e Tendências. XXXIX Encontro Nacional de Engenharia de Produção. São Paulo.

Bueno, Robson Elias. (2019). Procurement 4.0: Barreiras e Oportunidades na Indústria 4.0. Dissertação apresentada ao programa de Pós-Graduação em Engenharia de Produção da Universidade Paulista - UNIP para obtenção do título de Mestre em Engenharia de Produção. São Paulo: UNIP.

Grande, Márcia Mazzeo; Olhê, Enrico Arnaldo; Rodello, Ildeberto Aparecido; Gomes, Erasmo José. (2016). E-Procurement: Conceitos, Modelos e Fatores Críticos de Sucesso. Revista Eletrônica de Administração, vol. 22, núm. 3, Universidade Federal do Rio Grande do Sul. Porto Alegre. ISSN 1980-4164. 
Haddud, Abu. (2018). Procurement 4.0: factors influencing the digitisation of procurement and supply chains. Business Process Management Journal. Vol. 24. No. 4. pp. 965-984. ISSN 14637154.

DOI 10.1108/BPMJ-06-2017-0139

Ito, Christian; Santos, Fábio de Sousa. (2020). E-Procurement e Contratos inteligentes: desafios da modernização tecnológica da contratação pública no Brasil. International Journal of Digital Law - IJDL ano 01. n. 02. maio/agosto. ISSN 2675-7087. DOI: 10.47975/digital.law.vol.1.n.2 02.

Petronas. (2019). Desvendando a Indústria 4.0: Saiba Tudo Sobre a Nova Revolução Industrial. Kuala Lumpur: Blog Inovação Industrial/PETRONAS.

Pinto, Daniela Mary Terra Ferreira. Procurement 4.0: como a área de compras está se posicionando frente às novas tecnologias e a transformação digital? São Paulo: Pontifícia Universidade Católica de São Paulo, 2019. Disponível: https://www.pucsp.br/sites/default/files/download/efisus/Procurement_4.pdf. Acesso: 03/2021

Schreiber, Bernd; Janssen, Rolf; Weaver, Stephan; Peintner, Stefan. (2016). Global Procurement 4.0 in the digital world. Disponível em: https://www.adlittle.com/en/insights/viewpoints/procurement-40-digital-world. Acesso em: 20 out. 2020.

Schwab, Klaus. (2016). A Quarta Revolução Industrial. World Economic Forum. Tradução: Daniel Moreira Miranda. 1르로ão. São Paulo: Edipro, 2016.

Souza, Erick R. R. (2018). Entenda Sobre Indústria 4.0: A Quarta Revolução Industrial que Estamos Vivendo Hoje. e-book Kindle/Amazon. Disponível: https://www.amazon.com.br/kindle/dp/B07K2D49RL/ref=rdr_kindle_ext_eos_detail\#detailBull ets_feature_div. Acesso: 03/2021. 